\title{
Group B streptococcus colonization of pregnant women and their children observed on obstetric and neonatal wards of the University Hospital in Krakow, Poland
}

Correspondence

Piotr B. Heczko

mbheczko@cyf-kr.edu.pl

Received 24 April 2008

Accepted 1 October 2008

\author{
Magdalena Strus, ${ }^{1}$ Dorota Pawlik, ${ }^{2}$ Monika Brzychczy-Włoch, ${ }^{1}$ \\ Tomasz Gosiewski, ${ }^{1}$ Krzysztof Rytlewski, ${ }^{3}$ Ryszard Lauterbach ${ }^{2}$ \\ and Piotr B. Heczko ${ }^{1}$ \\ ${ }^{1}$ Chair of Microbiology, Jagiellonian University Medical College, Krakow, Poland \\ ${ }^{2}$ Department of Neonatology, Jagiellonian University Medical College, Krakow, Poland \\ ${ }^{3}$ Department of Obstetrics, Gynaecology and Oncology, Chair of Obstetrics and Gynaecology, \\ Jagiellonian University Medical College, Krakow, Poland
}

\section{INTRODUCTION}

Group B streptococcus (GBS; Streptococcus agalactiae) has been implicated as a leading cause of neonatal invasive infections. Many adults are asymptomatically colonized with GBS in the genital and gastrointestinal tracts but colonized pregnant women are at increased risk of premature delivery and perinatal transmission to their neonates (Schuchat, 2000). GBS has been the principal cause of sepsis and meningitis during the early and late onset of neonatal infection. It is generally accepted that a neonate acquires its microflora, including GBS, by aspiration of the infected amniotic fluid, by vertical

Abbreviations: CDC, Centers for Disease Control and Prevention; FISH, fluorescence in situ hybridization; GBS, group B streptococcus; $M L S_{B}$, macrolide-lincosamide-streptogramin $\mathrm{B}$; RAPD, random amplification of polymorphic DNA. transmission during labour and later on also from the hospital environment.

The systemic introduction of the appropriate antimicrobial drug after the onset of labour or rupture of membranes is highly effective in reducing neonatal colonization with GBS, and therefore in 1996 in the USA two consensus statement guidelines were issued on intrapartum prophylaxis (CDC, 1996; Schrag et al., 2002), which reduced early neonatal GBS infection to less than $50 \%$ of previous levels. The recommended laboratory techniques may be enhanced by applying rapid identification methods and, when susceptibility testing is indicated, by determining inducible clindamycin resistance in conjunction with screening (Welch \& Aldridge, 2005).

Epidemiological data on GBS-infected newborns indicate that the mean incidence rate of invasive GBS infection is 0.7/1000 live births (Trijbels-Smeulders et al., 2004). Quite 
recently, a marked increase in fatality among cases of invasive GBS infection was reported in Norway (Hajdu et al., 2006). Several recent reports from Polish hospitals also noted an increase of both colonization and infection rates of neonates with GBS (Kowalska et al., 2003; Galińska et al., 2001).

This study was arranged in order to check the actual rates of colonization of pregnant women and their children with GBS in a Polish university hospital using the standard laboratory method of GBS detection recommended by the Centers for Disease Control and Prevention (CDC) and to estimate the frequency of resistance of these cocci to macrolides and clindamycin. Routes of transmission of GBS were followed in some cases using molecular typing.

\section{METHODS}

Subjects and GBS cultures. Colonization with GBS was checked in 340 pregnant women living in the south-eastern region of Poland (Małopolska) and attending in- and outpatient clinics of the University Hospital in Cracow in the years 2004-2006. The study protocol was approved by the Bioethical Committee of Jagiellonian University (no. KBET/267/B/2002 from 27 June 2003).

Subjects belonged to two different groups. The first group was composed of 250 tested women with a normal pregnancy who were admitted for delivery to the Clinical Ward of the Gynaecology and Infertility Department, University Hospital in Cracow (ward A). They reported no infection that required antibiotic treatment during the third trimester. They were checked for GBS carriage according to the CDC procedure (Schrag et al., 2002). Briefly, rectovaginal swabs were placed in Amies transport medium and immediately delivered to the microbiology laboratory, where they were transferred to $1 \mathrm{ml}$ selective Todd-Hewitt broth supplemented with gentamicin $\left(8 \mu \mathrm{g} \mathrm{ml}^{-1}\right)$ and nalidixic acid $\left(15 \mu \mathrm{g} \mathrm{ml} \mathrm{m}^{-1}\right)$ and incubated for $18 \mathrm{~h}$ at $37^{\circ} \mathrm{C}$. Subcultures on Columbia blood agar with $5 \%$ sheep blood were made and incubated for $24 \mathrm{~h}$ at $37{ }^{\circ} \mathrm{C}$. Suspected colonies were then inoculated on GBS medium with serum, Bile Aesculin Azide medium and Columbia blood agar with $5 \%$ sheep blood to demonstrate the CAMP phenomenon (Christie et al., 1944). Identification of GBS was confirmed by latex agglutination assay. In doubtful cases, both phenotypic characterization using an API STREP kit (bioMérieux) and molecular identification based on a PCR procedure with Sag59 and Sag190 species-specific primers for S. agalactiae were applied (Ke et al., 2000).

The second group consisted of 90 women with complicated pregnancy admitted for delivery to the High Risk Pregnancy Unit of the Department of Gynaecology, Obstetrics and Oncology, University Hospital in Cracow (ward B), because of previous complications of pregnancy: hypertension during pregnancy/preeclampsia/eclampsia, diabetes mellitus, cardiac disease, threatened preterm labour and premature rupture of membranes. Materials were obtained and culture methods used were exactly as in the first group of patients.

Newborns of women in both groups were also tested for GBS colonization by taking swabs from the external ear and oral cavity during suction. The swabs were placed in Amies transport medium and then tested for GBS growth according to the CDC procedure, as described previously.

GBS strain characterization. All isolated and positively identified GBS strains were tested for their $M_{L} S_{B}$ resistance. The macrolide- resistance phenotypes of erythromycin-resistant GBS were determined by the disc diffusion method on Mueller-Hinton agar supplemented with $5 \%$ sheep blood using erythromycin $(15 \mu \mathrm{g})$ and clindamycin $(2 \mu \mathrm{g})$ discs. Blunting of the clindamycin inhibition zone proximal to the erythromycin disc indicated an inducible type of $\mathrm{MLS}_{\mathrm{B}}$ resistance (Desjardins et al., 2004). Resistance to both erythromycin and clindamycin indicated a constitutive type of $\mathrm{MLS}_{\mathrm{B}}$ resistance. Susceptibility to clindamycin with no blunting defined the $M$ phenotype (efflux mechanism).

GBS strains isolated from neonates from non-colonized mothers and from pairs of mothers and children delivered by Caesarean section before rupture of membranes were tested for their genetic relatedness using a PCR-RAPD (random amplification of polymorphic DNA) method according to Martinez et al. (2000) with the primer OPB17 (AGG GAA CGA G). Analysis of similarities was performed using the Molecular Analyst (Bio-Rad) computer program.

Application of the fluorescence in situ hybridization (FISH) method to detect GBS directly in vaginal swabs was checked using the $S$. agalactiae specific probe Saga 67 a/b (GTAAACACCCMTCAGCG) labelled with CY3 stain (Artz et al., 2003). Total DNA was counterstained with DAPI 50 (Sigma). The vaginal swabs for FISH were taken at the same time as those for culture from all women and kept frozen at $-70{ }^{\circ} \mathrm{C}$. After completing the culture procedure, swabs for FISH were randomly selected from those representing women colonized with GBS. The swabs were thawed and smeared on appropriate sterile glass plates (Super Frost Plus; Menzel-Glaser) and, after fixation and hybridization, observed for GBS fluorescence under a fluorescence microscope (Olympus PX51). The same swabs were placed in $1 \mathrm{ml}$ Todd-Hewitt broth, vortexed and serial 10-fold dilutions were then made using the same medium. One hundred microlitre aliquots of each dilution were placed on Columbia blood agar plates with $5 \%$ sheep blood and incubated at $37^{\circ} \mathrm{C}$ for $24 \mathrm{~h}$. Numbers of GBS cells (c.f.u. per swab) were then calculated using the standard viable count method. Statistical analysis of the obtained data was performed using a standard chi-square test.

\section{RESULTS AND DISCUSSION}

When comparing rates of GBS colonization in two populations of pregnant women using the procedure recommended by the CDC, we have shown that women with a complicated pregnancy had a higher colonization rate than women with a normal pregnancy, but the difference was not significant (Table 1). Women with a complicated pregnancy were more often colonized with GBS strains of the $\mathrm{MLS}_{\mathrm{B}}$ phenotype indicating constitutive resistance to macrolides and clindamycin than those with a normal pregnancy.

We have also checked the possibility of using a modern FISH technique to detect GBS directly in vaginal swabs (Fig. 1). As shown in Table 2, the usefulness of the method is limited by its sensitivity since it detects only GBS populations exceeding $5 \times 10^{3}$ per swab.

Rates of GBS colonization in neonates born from GBScolonized mothers varied from $26.7 \%$ to $35 \%$. Neonates born from mothers with a complicated pregnancy were more often colonized with GBS than those from mothers with a normal pregnancy (Table 3). This difference was, however, not significant. Two children of the former group were colonized with antibiotic-resistant GBS and infected 
Table 1. Comparison of GBS colonization rates in women with normal and complicated pregnancy

\begin{tabular}{|lcc|}
\hline & Groups of pregnant women \\
\cline { 2 - 3 } & With normal pregnancy & With complicated pregnancy \\
\hline $\begin{array}{l}\text { No. of tested women } \\
\text { No. of GBS-colonized women } \\
\text { No. of women colonized by GBS strains with MLS } \text { B }_{\mathrm{B}} \text { resistance } \\
\text { mechanism }\end{array}$ & 250 & 90 \\
\hline
\end{tabular}

by these bacteria. It should be noted that both neonates colonized with resistant GBS died because of generalized infections caused by GBS.

Further analysis revealed that 12 newborns ( 5 of them born in ward A and 7 born in ward B) were colonized with GBS although they had been born from mothers not colonized with GBS. The GBS strains isolated from these neonates were grouped according to wards and analysed for their relatedness (Fig. 2) using the PCR-RAPD method and computer analysis of similarity. Only two GBS strains (B126/C and B127/C) isolated from two children born on the same day from different mothers but in the same ward were identical. The remaining strains showed different degrees of similarity from 30 to $80 \%$. However, this analysis showed that GBS strains isolated from children hospitalized in two different wards located in the same building were also very closely related (B126/C and B127/C versus $\mathrm{A} 62 / \mathrm{C})$. This may suggest a horizontal transmission of GBS strains in the hospital setting.

Also, it appeared that neonates born from GBS-colonized mothers acquired the cocci despite being delivered by Caesarean section before rupture of membranes. The GBS strains isolated from them and their colonized mothers were compared using the PCR-RAPD method (Fig. 3). It appeared that GBS strains found in three out of four pairs of mothers and children showed identical profiles.

GBS colonization rates of women in our studies varied from $17.2 \%$ for those with a normal pregnancy to $20 \%$ for women with a complicated pregnancy. This value is much higher than the $6.6 \%$ rate reported from Greek hospitals (Tsolia et al., 2003) but corresponds to a recent report from German clinical centres where the rate in pregnant women was $16 \%$ (Brimil et al., 2006). According to Welch \& Aldridge (2005), GBS detection depends strongly on laboratory experience and this may explain the differences among various centres and countries. However, we believe that an increase in GBS colonization rates in Poland is a reflection of the recent changes in socio-economic status of the Polish population since the same trends are observed for other modern civilization-related diseases such as atopy and inflammatory bowel disease (Wiercinska-Drapalo et al., 2005).

FISH applied for direct detection of GBS colonization seems to be a very useful and rapid method since the test can be completed in $4-5 \mathrm{~h}$ after collecting swabs. Thus it may be quite useful in laboratory practice since it gives highly specific results in a shorter time than PCR and culture, but still it is limited by the need to have sufficient numbers of GBS cells per microscope field (approx. $5 \times 10^{3}$

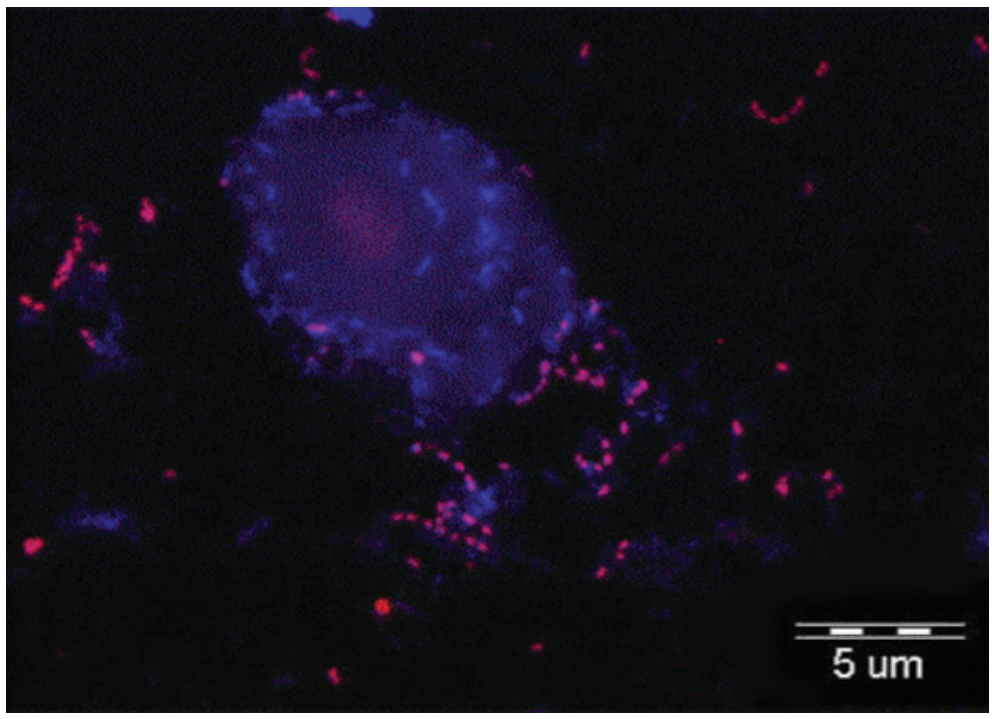

Fig. 1. Application of the FISH method for direct detection of GBS in a vaginal swab with $1.6 \times 10^{4}$ c.f.u. of the cocci. The swab was taken from woman no. A102, stained with species-specific probe Saga $67 \mathrm{a} / \mathrm{b}$ labelled with $\mathrm{CY} 3$ stain, counterstained with DAPI, and observed under an Olympus BX51 fluorescence microscope (magnification $\times 1000$ ). 
Table 2. Sensitivity of direct GBS detection in vaginal/ anorectal swabs by the FISH method in comparison to that of standard culture on blood agar plates

\begin{tabular}{|lcc|}
\hline GBS (c.f.u.) per swab & $\begin{array}{c}\text { Results of } \\
\text { FISH test }\end{array}$ & $\begin{array}{c}\text { No. of } \\
\text { tested samples }\end{array}$ \\
\hline$\leqslant 2.5 \times 10^{2}$ & - & 7 \\
$1.0-5.0 \times 10^{3}$ & $+/-$ & 5 \\
$0.5-5.0 \times 10^{4}$ & + & 11 \\
$0.5-5.0 \times 10^{5}$ & + & 6 \\
$\geqslant 2.5 \times 10^{6}$ & + & 1 \\
Total & & 30 \\
\hline
\end{tabular}

${ }^{*}-$, No S. agalactiae in the sample; $+/-$, questionable result;,$+ S$. agalactiae present in the sample.

per swab). Thus we have not confirmed data reported by Artz et al. (2003) on the sensitivity of FISH in comparison to culture since we have not been able to demonstrate low populations of GBS by FISH although they have been demonstrated by quantitative cultures. Although the CDC guidelines (CDC, 1996) do not distinguish different levels of GBS colonization, the FISH technique may have some practical implications in specific situations; for example, just before delivery, by eliminating light colonization. It has been demonstrated that the risk of GBS infection is much greater when the density of maternal colonization is higher than $10^{3}$ organisms (Benitz et al., 1999).

As has been shown in this study by comparison of GBS colonization rates in women with a normal pregnancy and those with a complicated pregnancy, the latter group was more often colonized with GBS although this difference
Table 3. Frequency of GBS detection in neonates born from women with a normal pregnancy and a complicated pregnancy

\begin{tabular}{|lcc|}
\hline No. of tested neonates & \multicolumn{2}{c|}{$\begin{array}{c}\text { No. of neonates born from women } \\
\text { with: }\end{array}$} \\
\cline { 2 - 3 } & $\begin{array}{c}\text { Normal pregnancy } \\
(\boldsymbol{n}=\mathbf{2 5 2})\end{array}$ & $\begin{array}{c}\text { Complicated } \\
\text { pregnancy }(\boldsymbol{n}=\mathbf{9 2})\end{array}$ \\
\hline $\begin{array}{c}\text { Total born from GBS- } \\
\text { colonized women }\end{array}$ & $45^{*}$ & $20^{\star}$ \\
$\begin{array}{l}\text { Colonized with GBS } \\
\text { Colonized by GBS strains } \\
\text { with MLS } \\
\text { mechanism }\end{array}$ & $12(26.7 \%)$ & $7(35 \%)$ \\
& 0 & $2 \dagger$ \\
\hline
\end{tabular}

${ }^{*}$ Including neonates from twin gestations.

$\dagger$ Fatal cases with invasive GBS infection documented by positive blood culture.

was not statistically significant. The higher rate of colonization of women with a complicated pregnancy with GBS expressing the MLS $_{\mathrm{B}}$ resistance mechanism may be related directly to their previous hospitalizations, increased numbers of different medical procedures and treatment regimens owing to their complicated pregnancies. This assumption seems to be augmented by the finding that children born from women with a complicated pregnancy were also more frequently colonized with the resistant GBS strains. In several studies, significant resistance to clindamycin and erythromycin has been noted (Chohan et al., 2006). Since in our study only a few GBS strains with the $\mathrm{MLS}_{\mathrm{B}}$ resistance phenotype were found, it is very difficult to speculate whether any substantial increase in numbers of

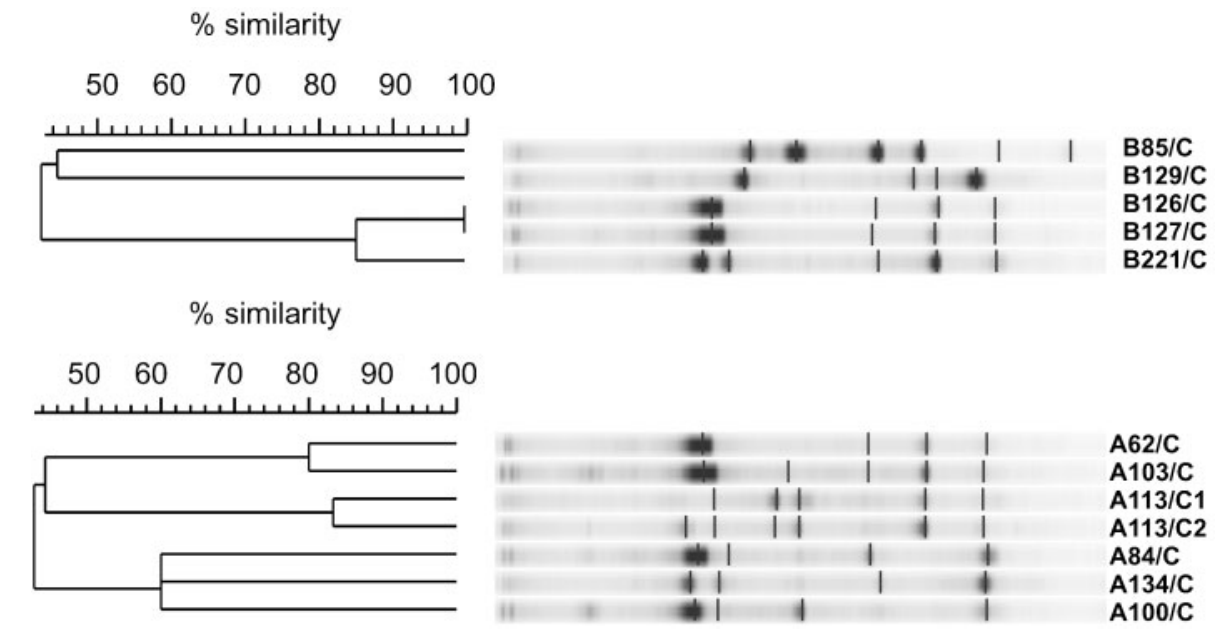

Fig. 2. Percentages of similarity, calculated using the Molecular Analyst version 1.6 (Bio-Rad) computer program, among GBS strains isolated from neonates delivered from non-colonized mothers in two different hospital wards, determined by the PCRRAPD method. Codes of strain numbers: ward (A, Department of Obstetrics, University Hospital in Cracow; B, Department of Gestation Pathology, University Hospital in Cracow)/consecutive number/child (C); C1 and C2 are twins. 


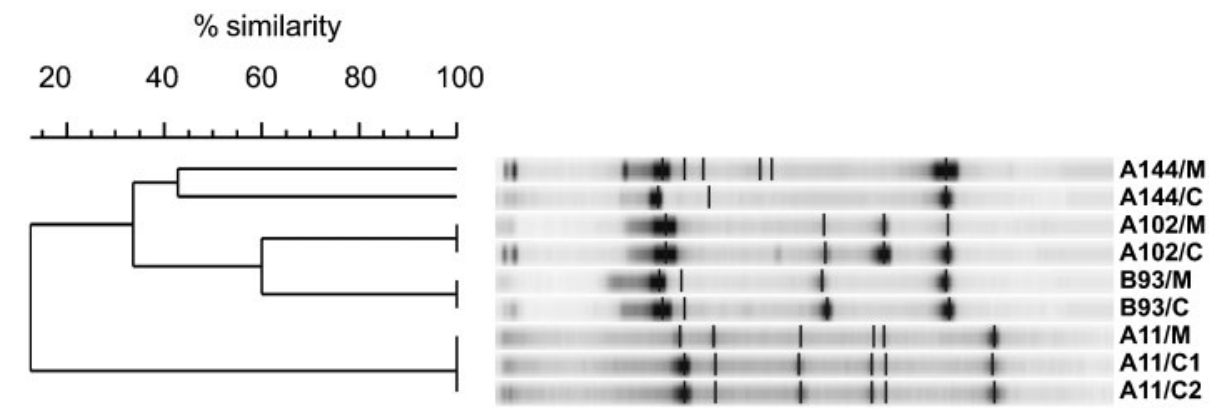

Fig. 3. Percentages of similarity, calculated using the Molecular Analyst version 1.6 (Bio-Rad) computer program, between pairs of GBS strains isolated from mothers and their newborns delivered by Caesarean section before rupture of membranes, determined by the PCR-RAPD method. Codes of strain numbers: ward (A, Department of Obstetrics, University Hospital in Cracow; B, Department of Gestation Pathology, University Hospital in Cracow)/consecutive number/mother (M) or child (C); $\mathrm{C} 1$ and $\mathrm{C} 2$ are twins.

resistant GBS isolated from pregnant women and their children has occurred recently in our hospital.

It is of interest that GBS can be easily transferred to a neonate. We have demonstrated on the basis of molecular strain identification that identical GBS strains were found in mothers and their newborns after Caesarean section done before rupture of the membranes. It is commonly believed that GBS colonization of newborns born by Caesarean section is delayed and rare (Rotimi et al., 1985) and that acquisition of the bacterial flora by newborns born by Caesarean section before rupture of membranes is generally impaired (Fanaro et al., 2003).

Conversely, other neonates born from mothers not colonized with GBS acquired these cocci from the hospital environment. Such a mechanism of early-onset infection in neonates caused by hospital environment-acquired GBS has recently been demonstrated by Puopolo et al. (2005).

Our study clearly indicates that rates of GBS colonization among pregnant women and neonates in a university hospital in Poland have reached levels comparable to those reported in other European clinical centres and may support the suggestion that a common European consensus on prevention policies should now be established (de la Rosa Fraile et al., 2001).

\section{ACKNOWLEDGEMENTS}

We thank Dr Piotr Kochan, MD for language consultation and adapting of the manuscript to requirements, and Mrs Helena Machlarz for her excellent assistance in organizing clinical work. This study was supported by grant no. 3 PO5E 08425 from the Polish Ministry of Science and Higher Education.

\section{REFERENCES}

Artz, L. A., Kempf, V. A. \& Autenrieth, I. B. (2003). Rapid screening for Streptococcus agalactiae in vaginal specimens of pregnant women by fluorescent in situ hybridization. J Clin Microbiol 41, 2170-2173.
Benitz, W. E., Gould, J. B. \& Druzin, M. L. (1999). Risk factors for early-onset group B streptococcal sepsis: estimation of odds ratios by critical literature review. Pediatrics 103, e77.

Brimil, N., Barthell, E., Heindrichs, U., Kuhn, M., Lutticken, R. \& Spellerberg, B. (2006). Epidemiology of Streptococcus agalactiae colonization in Germany. Int J Med Microbiol 296, 39-44.

CDC (1996). Prevention of perinatal group B streptococcal disease: a public health perspective. MMWR 45, 1-24.

Chohan, L., Hollier, L. M., Bishop, K. \& Kilpatrick, C. C. (2006). Patterns of antibiotic resistance among group B streptococcus isolates: 2001-2004. Infect Dis Obstet Gynecol 2006, 57492.

Christie, R., Atkins, N. E. \& Munch-Peterson, E. (1944). A note on a lytic phenomenon shown by group B streptococci. Aust J Exp Biol 22, 197-200.

de la Rosa Fraile, M., Cabero, L., Andreu, A. \& Rao, G. G. (2001). Prevention of group B streptococcal neonatal disease: a plea for a European consensus. Clin Microbiol Infect 7, 25-27.

Desjardins, M., Delgaty, K. L., Ramotar, K., Seetaram, C. \& Toye, B. (2004). Prevalence and mechanisms of erythromycin resistance in group A and group B streptococcus: implications for reporting susceptibility results. J Clin Microbiol 42, 5620-5623.

Fanaro, S., Chierici, R., Guerrini, P. \& Vigi, V. (2003). Intestinal microflora in early infancy: composition and development. Acta Paediatr Suppl 91, 48-55.

Galińska, A., Szponar, M. \& Bohdan, Z. (2001). B Streptococcus and other pathogenic bacteria colonization in hospitals in the Gdansk Metro Area. Przegl Epidemiol 55 (Suppl.), 55-61.

Hajdu, A., Blystad, H., Høiby, E. A., Klouman, E., Schimmer, B. \& Nygård, K. (2006). Unexpected increase in case fatality of invasive group B streptococcal infections in infants in Norway, January-July 2006. Euro Surveill 11, E060727.2.

Ke, D., Menard, C., Picard, F. J., Boissinot, M., Ouellette, M., Roy, P. H. \& Bergeron, M. G. (2000). Development of conventional and real-time PCR assays for the rapid detection of group B streptococci. Clin Chem 46, 324-331.

Kowalska, B., Niemiec, K. T., Drejewicz, H., Polak, K., Kubik, P., Elmidaoui, A., Gierowska-Bogusz, B. \& Jaczynska, R. (2003). Prevalence of group B streptococcal colonization in pregnant women and their newborns based on the results of examination of patients in the Obstetric and Gynecology Department of the National Research Institute of Mother and Child - a pilot study. Ginekol Pol 74, 1223-1227. 
Martinez, G., Harel, J., Higgins, R., Lacouture, S., Daignault, D. \& Gottschalk, M. (2000). Characterization of Streptococcus agalactiae isolates of bovine and human origin by randomly amplified polymorphic DNA analysis. J Clin Microbiol 38, 71-78.

Puopolo, K. M., Madoff, L. C. \& Eichenwald, E. C. (2005). Early-onset group B streptococcal disease in the era of maternal screening. Pediatrics 115, 1240-1246.

Rotimi, V. O., Olowe, S. A. \& Ahmed, I. (1985). The development of bacterial flora of premature neonates. J Hyg (Lond) 94, 309-318.

Schrag, S., Gorwitz, R., Fultz-Butts, K. \& Schuchat, A. (2002). Prevention of perinatal group B streptococcal disease. Revised guidelines from CDC. MMWR Recomm Rep 51, 1-22.

Schuchat, A. (2000). Neonatal group B streptococcal disease: screening and prevention. N Engl J Med 343, 209-210.
Trijbels-Smeulders, M. A., Kollee, L. A., Adriaansee, A. H., Kimpen, J. L. \& Gerards, L. J. (2004). Neonatal group B streptococcal infection: incidence and strategies for prevention in Europe. Pediatr Infect Dis J 23, 172-173.

Tsolia, M., Psoma, M., Gavrili, S., Petrochilou, V., Michalas, S., Legakis, N. \& Karpathios, T. (2003). Group B streptococcus colonization of Greek pregnant women and neonates: prevalence, risk factors and serotypes. Clin Microbiol Infect 9, 832-838.

Welch, D. F. \& Aldridge, K. E. (2005). Optimizing the rapid and accurate detection of group B streptococci from antepartum cultures. Infect Med 22, 133-137.

Wiercinska-Drapalo, A., Jaroszewicz, J., Flisiak, R. \& Prokopowicz, D. (2005). Epidemiological characteristics of inflammatory bowel disease in North-Eastern Poland. World J Gastroenterol 11, 2630-2633. 